\title{
Sarcoidosis at the Pulmonary Medicine Department of a tertiary care hospital in Mumbai. Our experience and The New Modified Criteria Clinical Radiological Physiological (TNMC CRP) score for sarcoidosis: A novel proposition to assess the functional status
}

\author{
Ketaki Utpat, Chinnu Sasikumar, Unnati Desai, Jyotsna M. Joshi \\ Department of Pulmonary Medicine, Topiwala National Medical College and BYL Nair Hospital, Mumbai, India
}

\begin{abstract}
Sarcoidosis is a multisystemic granulomatous disease most commonly involving the pulmonary system and having a myriad of manifestations. However literature is scanty pertaining to the profile and scoring system in sarcoidosis. This study was undertaken
\end{abstract}

\begin{abstract}
Correspondence: Dr. Unnati Desai, Associate Professor and Incharge, Department of Pulmonary Medicine, 2nd floor, OPD bldg, Topiwala National Medical College and BYL Nair Hospital, Mumbai 400008, India. Tel.+91.022.23027642/43. E-mail: unnati_desai82@yahoo.co.in

Keywords: Sarcoidosis; TNMC CRP score; 6MWD.

Authors' contributions: KU, concepts, design, definition of intellectual content, literature search, data acquisition and analysis, manuscript preparation, editing and review; CS, literature search, data acquisition and analysis, statistical analysis, manuscript preparation and editing; UD, JMJ, definition of intellectual content, concept, design, manuscript editing and review. All the authors have read and approved the final version of the manuscript and agreed to be accountable for all aspects of the work.
\end{abstract}

Conflict of interest: The authors declare that they have no competing interests, and all authors confirm accuracy.

Availability of data and materials: The datasets used and/or analyzed during the current study are available from the corresponding author on reasonable request.

Ethics approval: This was a retrospective study undertaken with Institutional Ethics Committee permission at the Pulmonary Medicine Department of a tertiary care hospital in Mumbai.

Patient consent for publication: Not applicable.

Received for publication: 9 October 2020.

Accepted for publication: 11 December 2020.

${ }^{\circ}$ Copyright: the Author(s), 2021

Licensee PAGEPress, Italy

Monaldi Archives for Chest Disease 2021; 91:1636

doi: 10.4081/monaldi.2021.1636

This article is distributed under the terms of the Creative Commons Attribution Noncommercial License (by-nc 4.0) which permits any noncommercial use, distribution, and reproduction in any medium, provided the original author(s) and source are credited. to understand the profile of sarcoidosis and an endeavor to assess the functional status with a simplified scoring system. This was an observational study undertaken in the department of Pulmonary Medicine at a tertiary care. The profile of these patients was studied in terms of clinical features, radiological findings, the New Modified Criteria Clinical Radiological Physiological (TNMC CRP) score, six-minute walk distance (6MWD), spirometry, arterial blood gas parameters, serum angiotensin converting enzyme (ACE) levels and tissue biopsy histopathology. The 68 patients included 41 women and 27 men with a mean age of 42.7 years. They comprised of 18 (27\%), 39 (57\%), 4 (6\%), 7 (10\%) cases of stage 1, 2, 3, 4 sarcoidosis respectively. Most common presenting symptom and sign was progressive dyspnea 49 (72\%), and peripheral lymphadenopathy 15 (22\%). Serum ACE was elevated in 57 (83\%). The average 6MWD was 360 meters. Most common high resolution computed tomography (HRCT) finding was mediastinal lymphadenopathy and peri-bronchovascular nodules. Spirometry was restrictive abnormality in $48(96 \%)$ patients. Evidence of pulmonary hypertension $(\mathrm{PH})$ was present in $32(47 \%)$ patients. Tissue diagnosis revealed granulomatous inflammation in 51 biopsies with a transbronchial lung biopsy (TBLB) yield of $62 \%$. The average TNMC CRP score was 5. There was a positive correlation between this score and 6MWD which was statistically significant. The score correlated with the functional status. Diagnosis of sarcoidosis warrants a comprehensive and multimodality approach. HRCT and tissue biopsy are the most important diagnostic armamentariums. Modified simplified scores help assess the functional status of the disease.

\section{Introduction}

Sarcoidosis belongs to a large family of disorders that share granuloma formation as common denominator [1]. Sarcoidal granulomas can involve any organ. Clinical sarcoidosis is manifested as intrathoracic lymph node enlargement, pulmonary involvement, skin or ocular signs and symptoms, or some combination of these findings in most cases [2]. Diagnosis is established by a multidisciplinary approach by a combination of clinico-radiological features supported by histopathological evidence of noncaseating granulomas after exclusion of other known causes. Sarcoidosis is reported less commonly in India and this has been attributed to factors such as its resemblance to tuberculosis [3], lack of screening programs and lack of awareness amongst physicians and pathologists regarding the disease. However, sarcoidosis is not a rare disease in India as initially thought. This is highlight- 
ed in various studies [4]. About $90 \%$ of patients have pulmonary lesions and $60 \%$ of them have respiratory complains. Extra pulmonary manifestations of sarcoidosis include involvement of skin, eye, heart, nervous system, endocrine and exocrine glands, gastrointestinal, genitourinary, hematological and immunological systems, bones, muscle and joints. There is significant heterogeneity in the onset, clinical course, prognosis and complications of the disease. Also there is scarcity of simplified scores that can serve as surrogate markers of disease severity and aid prognostication. Hence, we conducted this study to understand the spectrum of clinico-radiological manifestations of sarcoidosis and to propose a simple score (a modification of the existing CRP score) to assess the functional status.

\section{Materials and Methods}

This was a retrospective study undertaken with Institutional Ethics Committee permission at the Pulmonary Medicine Department of a tertiary care hospital in Mumbai. We included 68 consecutive cases of sarcoidosis over a three-year period. Diagnosis of sarcoidosis was established on the basis of clinicoradiological features supported by histopathological evidence of non-caseating granulomas after exclusion of other known causes. Demography details were noted. Symptoms including cough, dyspnea, fever, chest pain, and skin, ocular and joint complaints were noted. Detailed general and systemic examination was performed. Post exercise desaturation, 6-min walk test distance (6MWD), and arterial blood gas analysis (ABG) were noted. Baseline hematological and biochemical tests and Mantoux test were performed. Radiological tests in form of chest X-ray (CXR) and high resolution computerized tomography (HRCT) of thorax were done. Spirometry was done to assess the forced expiratory volume in first second (FEV1), forced vital capacity (FVC) and FEV1/FVC ratio. Serum Angiotensin converting enzyme (ACE) level and wherever possible 18 fluoro-deoxy glucose positron emission tomography (FDG PET) and gallium scan reports were noted. Histopathological diagnoses were obtained by biopsy or fine needle aspiration cytology of the organ system involved. The various sites biopsied were transbronchial lung biopsy (TBLB), peripheral lymph nodes, mediastinal lymph nodes, skin, thyroid and Mantoux site. Clinical radiological physiological (CRP) score is a scoring system that can be used in patients with sarcoidosis to assess the functional status. This score incorporates parameters like clinical symptoms, radiographic stage and physiological tests. CRP score is calculated as the sum of all the scores. Score of 1 is assigned for individual symptoms such as cough, dyspnea and weight loss. Radiological staging is scored as $0,1,2,3,4$ points for stage 0,1 , 2,3 and 4, respectively. Score of 1 each is assigned for the presence of obstructive, restrictive or mixed defects in spirometry, reduced diffusing capacity of the lungs for carbon monoxide (DLCO) and partial pressure of oxygen $\left(\mathrm{PaO}_{2}\right)<70 \mathrm{mmHg}$ [5]. Though of salient utility this score may have certain shortcomings. One of it involves the need to perform a sophisticated lung function assessment with DLCO which may be a hindrance in resource limited settings. Hence in an attempt to overcome this obstacle, we formulated a new score, TNMC CRP Score replacing DLCO test with ACE level. The rationale behind this was that DLCO is difficult to perform test and may not be readily available in resource limited settings. On the other hand, serum ACE levels are comparatively easier to perform and available in most standardized laboratories. The New Modified Criteria Clinical Radiological
Physiological (TNMC CRP) score replaced the parameter "reduced DLCO values" with "elevated S.ACE levels". The abbreviation denoted the purpose of the test and the abbreviation of our institute, too. The data was analyzed and was presented in the form of percentage and frequency.

\section{Results}

Sixty-eight patients were included in the study. They comprised of $18(27 \%), 39(57 \%), 4(6 \%), 7(10 \%)$ cases of stage 1,2 , 3, 4 sarcoidosis respectively. Most of the patients (40) belonged to the age bracket of 31-50 years. Mean age was 42.7 years. Our study included 41 women and 27 men suggesting a female preponderance. Most common symptoms were progressive dyspnea in $49(72 \%)$ followed by dry cough in $50(74 \%)$, productive cough in $24(36 \%)$, fever in $21(32 \%)$, chest pain in $8(12 \%)$ and hemoptysis in $4(5.88 \%)$. Five patients were asymptomatic cases of type 1 sarcoidosis picked up incidentally on routine examination. Most common extra pulmonary symptoms observed were ophthalmic in nature in $10(16 \%)$. The complaints of red eye, blurred vision and reduced vision were predominantly reported. Miscellaneous other extrapulmonary symptoms were hoarseness of voice, skin rash, dysphagia, joint pain, altered bowel habits, generalized weakness, myalgia and menstrual abnormalities in 1 patient each respectively. On general examination, the most common finding was the presence of peripheral lymphadenopathy in $15(22 \%)$. There were 6,4 , 1 patients of cervical, axillary and dual inguinal with axillary lymphadenopathy, respectively. Second most common finding was presence of clubbing seen in $13(20 \%)$ cases. In $39(58 \%)$ patients, the general examination was normal. The findings of systemic examination were unremarkable in $43(64 \%)$. Twenty-one $(32 \%)$ showed presence of bibasilar crackles, $2(4 \%)$ had bronchial breath sounds and $4(6 \%)$ had reduced breath sounds on the right side. All 4 patients with reduced breath sounds had hydropneumothoraxes. Serum ACE levels were elevated in 36(72\%). Highest level of ACE level was $310 \mathrm{u} / \mathrm{l}$. Lowest level encountered was $15 \mathrm{u} / \mathrm{l}$. The average ACE level encountered in the study was $91.6 \mathrm{u} / \mathrm{l}$. The Mantoux test was done in all patients. It was negative in 44 and positive in 6 patients. $6 \mathrm{MWD}$ and $\mathrm{ABG}$ were done in all except type I sarcoidosis. The average 6MWD was $360 \mathrm{~m}$.

Chest radiograph findings suggested type I disease in 19 (28\%), type 2 in $28(41 \%)$ and type 3 in 21 (31\%). Miscellaneous CXR findings were hydropneumothoraxes (3), collapse (1), fibrosis (2), fissural thickening (1), pneumothorax (1) and pleural plaques (1). HRCT thorax was done in all patients. After incorporating the findings of HRCT thorax, the frequency of various types of sarcoidosis in the study changed to $18(27 \%), 39(57 \%), 4(6 \%)$, $7(10 \%)$ for type $1,2,3,4$, respectively. Thus, HRCT thorax played a very important role in diagnosis and also staging of sarcoidosis. Most common HRCT finding was mediastinal lymphadenopathy (64/68), peri-fissural nodules (50/68), peri-lymphatic nodules $(50 / 68)$ and peri-bronchovascular nodules $(50 / 68)$. Other frequent findings included air trapping, consolidation and ground glass attenuation. Fluorodeoxyglucose positron emission tomography (FDG PET) scan reports were available in 32 patients. In 8, the only FDG avid structures were the mediastinal lymphnodes (all 7 of type 1 sarcoidosis). Twenty-four patients had both lung and mediastinal lymph nodal FDG uptake (21 of type 2 sarcoidosis and 2 of type 4 sarcoidosis). One patient of type 3 sarcoidosis had isolated FDG uptake in the lung. Gallium scan reports were available in 7 patients. In 4 cases, the structure with intense gallium uptake 
was mediastinal lymph nodes. Two had uptake in lung and one patient had no uptake on gallium scan. Spirometry was done in all patients. It was found to be restrictive in $48(70 \%)$, obstructive in $2(3 \%)$ and normal in $18(27 \%)$. ECG was normal in 44, however ECG abnormalities were detected in 6 patients. Most commonly encountered ECG finding was right bundle branch block. Twodimensional echocardiography (2D ECHO) showed that 23 (34\%), $2(3 \%), 7(10 \%)$ patients had mild, moderate and severe pulmonary hypertension $(\mathrm{PH})$, respectively.

Tissue diagnosis was done in all patients. Therefore, the total number of biopsies done in this study was 68 (Table 1). Granulomatous inflammation was seen in 51 biopsies (Table 2). In the rest, the diagnosis was established with clinico-radiological and serology correlation. The different tissue biopsy sites were TBLB (26), peripheral lymphnode (10), mediastinal lymph node (9), skin (3), Mantoux site (2), thyroid (1) (Table 1). Out of the 42 TBLBs performed, $26(62 \%)$ showed the evidence of granuloma on histopathological examination. Distribution of granulomas diagnosis was $17 / 18,28 / 39,2 / 4$ 4/7 for type 1, 2, 3 and 4 sarcoidosis, respectively. Thirty-eight patients enrolled in this study had concomitant presence of other comorbidities. The most common comorbidities found amongst this study population were gastroesophageal reflux disease (GERD) (21), hypertension (14), osteoporosis (14), OSAS (11), diabetes mellitus (11) and psychiatric illness in the form of anxiety or depression (6). Extrapulmonary involvement was seen in 12 patients. These included 2 cases with thyroid involvement, 10 cases with uveitis and one patient with renal calculi.

TNMC CRP score was calculated in all patients. In view of the unavailability of DLCO gas mixtures at times, the DLCO measurement was difficult at our centre. To overcome this problem, we replaced "DLCO reduction" scoring parameter with "elevated Serum ACE levels". The average TNMC CRP score was 5. The functional parameters in these patients were correlated with TNMC CRPS score. The 6MWD correlated with TNMC CRP score and the

Table 1. Various biopsy sites.

\begin{tabular}{lcc} 
Site of biopsy & Total & Granuloma obtained \\
Transbronchial lung biopsy & 42 & 26 \\
Peripheral lymph nodes & 10 & 10 \\
\hline Mediastinal lymph nodes & 10 & 9 \\
Skin & 3 & 3 \\
\hline Thyroid & 1 & 1 \\
Mantoux site biopsy & 2 & 2 \\
\hline Total & 68 & 51 \\
\hline
\end{tabular}

Table 2. Table showing the distribution of biopsied patients with granulomatous inflammation.

\begin{tabular}{lcc} 
Sarcoidosis & $\begin{array}{c}\text { Total patients } \\
\text { Total number of biopsies with } \\
\text { granulomatous inflammation }\end{array}$ \\
I & 18 & 17 \\
II & 39 & 28 \\
\hline III & 4 & 2 \\
IV & 7 & 4 \\
\hline Total & & 51 \\
\hline
\end{tabular}

correlation was found to be statistically significant with a $\mathrm{P}$ value $<0.0001$ based on Pearson $r$ coefficient with $r$ value -0.691 and $95 \%$ confidence interval -0.798 to -0.542 . 6MWD had positive correlation with forced vital capacity (FVC) when calculated with pearson coefficient with $\mathrm{P}$ value $<0.0001$ and $\mathrm{r}$ value 0.4993 . Thus, indirectly correlating with the TNMC-CRP score. Pulmonary hypertension $(\mathrm{PH})$ had positive correlation with modified TNMC CRP score with $\mathrm{p}$ value of $<0.0001$ and $\mathrm{r}$ value 0.5724 . The TNMC CRP score thus correlated well with the functional status.

\section{Discussion}

This was a retrospective observational study. It enrolled 68 patients of sarcoidosis. The patient group showed a slight female preponderance. As with many inflammatory diseases, sarcoidosis affected women more than men [6]. Most of the patients enrolled in this study belonged to the age group of 31 yrs to $50 \mathrm{yrs}$. Sarcoidosis usually develops before the age of 50 years, with the incidence peaking at 20 to 39 years. In a north Indian study, the average age at presentation was 43 years [6]. Studies from western countries report that more than $70 \%$ patients with sarcoidosis were of less than 40 years of age. Similar observations had been made in other Indian studies $[7,8]$. Dry cough was the commonest symptom in our study followed by progressive dyspnea. Similar findings were reported in an Indian study done by Sharma et al., in which the frequency of dry cough $(83 \%)$ was more than dyspnea (61\%) [8]. Fever was found in $32 \%$ of our patients. On the contrary, the incidence of fever documented in a study done in Indian population was higher $56 \%$ [9]. The most common non respiratory complaint that the patients had was ophthalmic in $10(16 \%)$ patients. This was consistent with one of the review articles suggesting eye involvement in 10-25\% cases [10]. Most common general examination finding in our study population was peripheral lymphadenopathy $(22 \%)$, commonest involved were cervical lymph nodes. Yanardag et al. reported an incidence of $14.5 \%$ for peripheral lymphadenopathy in sarcoidosis and the most commonly encountered lymphadenopathies were cervical and supraclavicular [11]. Clubbing was encountered in $13(20 \%)$ of our cases. Clubbing was a rare finding and usually seen in advanced fibrotic stage [12]. Respiratory system examination was unremarkable in around $64 \%$ of our study population and bilateral basal crackles were heard in $32 \%$ of patients. In a study done by Kumar et al. crackles were appreciated in $49.3 \%$ of patients [6]. In our study the serum ACE levels where elevated in $83 \%$. In a case control study done to assess the effectiveness of ACE levels in diagnosis and regulating therapy in sarcoidosis, they were elevated in about 15 of 17 patients [13].

CXR classified the patients as per the Scadding classification as $28 \%$ to stage $1,41 \%$ stage 2 , and $31 \%$ stage 3 . The ACCESS study [14] found that the $8 \%$ of the patients had stage $0,40 \%$ stage $1,36 \%$ stage $2,10 \%$ stage 3 , and 5\% stage 4 . CXR helped to diagnose certain complications like pneumothorax. After incorporating the HRCT thorax in diagnosis, the frequency of various types of sarcoidosis in our study changed. Thus it played a very important role in diagnosis and was more accurate as compared with CXR. Fatih and co-workers encountered a similar finding [15]. Mediastinal lymphadenopathy was the most common finding with or without lung involvement. Mediastinal lymph nodes were found in $94 \%$ of the study population. As per a review article on imaging features of sarcoidosis, $80 \%$ of sarcoidosis cases had mediastinal lymphadenopathy [16]. Most common group of mediastinal lymph 
nodes to be encountered in our study was hilar lymph node involvement. In most instances except in 3 cases, hilar lymphadenopathy was bilateral. The next most common finding on HRCT was the presence of peri-bronchovascular nodules $(73 \%)$ and peri-fissural nodules (73\%). In an HRCT based study of 18 biopsy proven cases of sarcoidosis, fourteen patients had predominant nodular opacities [17]. A study on FDG PET in sarcoidosis concurred with our finding of mediastinal lymph nodes being the commonest FDG avid structure [18]. The most common abnormality on spirometry was restrictive lung functions seen in $70 \%$ of the patients. A retrospective study from Brazil reported restrictive abnormality in $23 \%$ of the study population. This difference could be attributed to a larger proportion of type 2 and 3 Sarcoidosis cases in our study compared to the Brazilian study [19]. ECG abnormalities were seen in $8.9 \%$. In a Caucasian study on pulmonary sarcoidosis, an abnormal ECG was recorded in $16.3 \%$ patients [20]. In our study most of the patients had mild PH. In a retrospective evaluation of 106 patients with sarcoidosis, Sulica et $a l$. found that $51 \%$ of the patients with a pre-existing clinical indication for performing echocardiography had evidence of $\mathrm{PH}$ by transthoracic echocardiography [21].

We pursued for tissue diagnosis in all patients as histopathological demonstration is the most convincing and definitive mode of diagnosis [22]. Granulomatous inflammation was found in 51 biopsies (75\%). In rest the diagnosis was established with clinicoradiological and serology correlation. Most common method of tissue diagnosis was TBLB. Out of the 42 TBLBs performed, 26 $(62 \%)$ showed the evidence of granuloma on histopathological examination. Thus TBLB served as an extremely useful tool for the definitive diagnosis and could avoid the need for more invasive tissue diagnosis like a thoracoscopic or an open lung biopsy. Thirtyfive of our patients had concomitant presence of other comorbidities with GERD being the most common. A study done to assess the prevalence of GERD in interstitial lung diseases reported $70 \%$ of the patients with sarcoidosis to have GERD [23]. In a study for comorbid illnesses and chest radiographic severity in African American sarcoidosis patients, around $15 \%$ of the patients had GERD [24]. Another comorbidity associated with sarcoidosis was systemic hypertension. In a large cohort patient analysis, the incidence of systemic hypertension was $22.4 \%$ [25]. Eleven of our patients $(16 \%)$ had obstructive sleep apnea. In a study done by Turner and co-workers on sleep apnea in sarcoidosis, 14/83 where sleep apneic [26]. Contrary to our study, others have shown a higher percentage of extrapulmonary involvement. In a study, done in Turkey the percentage of extrapulmonary involvement was seen in $40 \%$ of the patients [27]. Ophthalmological evaluation was done in all patients and was normal in all except 10 patients. In a Chinese study, 60 patients with biopsy-proven sarcoidosis had uveitis [28]. The ATS sarcoidosis guidelines also recommend a baseline eye evaluation irrespective of symptoms [29]. They also recommend baseline serum creatinine, serum alkaline phosphatase, serum calcium and electrocardiogram to rule out extrathoracic manifestations of sarcoidosis [29].

A chronic lung disease with extrapulmonary manifestations like sarcoidosis needs a multifaceted assessment involving clinical, radiological and physiological assessment. This plays a vital role in the disease severity staging and prognostication. CRP score is a scoring system that was firstly used in IPF to predict the severity of the pathologic process and functional impairment. It was later employed in sarcoidosis to assess the functional status. This score incorporates parameters like clinical symptoms, radiographic stage and physiological tests. CRP score is calculated as the sum of all the scores. Score of 1 each is assigned for individual symptoms, such as cough, dyspnea, and weight loss. Radiological staging is scored as $0,1,2,3,4$ points for stage $0,1,2,3$, and 4 , respectively. Score of 1 each assigned for the presence of obstructive, restrictive, or mixed defects in spirometry, reduced DLCO and partial pressure of oxygen $\left(\mathrm{PaO}_{2}\right)<70 \mathrm{mmHg}^{5}$. In a retrospective study on 26 patients diagnosed with pulmonary sarcoidosis the 6MWD inversely correlated with the CRP score $(\rho=-0.364, p=0.047)$ and positively correlated with forced vital capacity (FVC) $\%(\rho=0.407$, $\mathrm{p}=0.039)^{5}$. Though of salient utility this score may have certain shortcomings. One of it involves the need to perform a sophisticated lung function assessment with DLCO which may be a hindrance in resource limited settings. Hence in an attempt to overcome this obstacle, we formulated a new score, TNMC CRP Score replacing DLCO test with ACE level. The rationale behind this was that DLCO is difficult to perform test and may not be readily available in resource limited settings. On the other hand, serum ACE levels are comparatively easier to perform and available in most standardized laboratories. The role of CRP was to assess the functional status. In diseases like Sarcoidosis serological markers like Serum ACE levels correlated and reflected well upon the functional status of the disease. The TNMC CRP was thus assessed with functional parameters. The 6MWD correlated inversely with TNMC CRP score with a statistically significant correlation with a $\mathrm{P}$ value $<0.0001$ based on Pearson $\mathrm{r}$ coefficient with $\mathrm{r}$ value -0.691 and $95 \%$ (confidence interval -0.798 to -0.542 ). 6MWD also had positive correlation with FVC when calculated with Pearson coefficient with $p$ value $<0.0001$ and $r$ value 0.4993 similar to the study done by Esam et al. [5]. Thus, indirectly correlating with FVC. The TNMC CRP score correlated well with the functional status of the disease. Hence, we propose that the TNMC CRP score may be considered as a possible alternative to assess the functional status of Sarcoidosis particularly in resource limited settings where facilities for DLCO measurement may not be available. However studies with larger sample size are required to endorse our results.

Our study thus stands noteworthy from various aspects. It is a comprehensive assessment of profile of Sarcoidosis involving a good sample size on a background of very limited data available on this disease which shows a good response to therapy. Our study also endeavors to assess the functional status in these patients with the aid of a novel scoring system which can be employed in resource limited settings, too.

\section{References}

1. Baughman RP, Lower EE, du Bois RM. Sarcoidosis. Lancet 2003;361:1111-8.

2. Iannuzzi MC, Rybicki BA, Teirstein AS. Sarcoidosis. N Engl J Med 2007;357:2153-65.

3. Nunes H, Soler P, Valeyre D. Pulmonary sarcoidosis. Allergy 2005;60:565-82.

4. Sharma SK, Mohan A. Sarcoidosis in India: Not so rare! J Indian Acad Clin Med 2004;5:12-21.

5. Alhamad EH. The six-minute walk test in patients with pulmonary sarcoidosis. Ann Thorac Med 2009;4:60-4.

6. Kumar R, Goel N, Gaur SN. Sarcoidosis in north Indian population: A retrospective study. Indian $\mathrm{J}$ Chest Dis All Sci 2012;54:99-104.

7. Jindal SK, Gupta D, Aggarwal AN. Sarcoidosis in developing countries. Curr Opin Pulm Med 2000;6:448-54.

8. Sharma SK, Mohan A, Guleria JS. Clinical characteristics, pulmonary function abnormalities and outcome of prednisolone 
treatment in 106 patients with sarcoidosis. J Assoc Physicians India 2001;49:697-704

9. Behbehani N, JayKrishnan B, Khadadah M, et al. Clinical presentation of sarcoidosis in a mixed population in the middle east. Respir Med 2007;101:2284-8.

10. Nunes H, Bouvry D, Soler P, Valeyre D. Sarcoidosis. Orphanet J Rare Dis 2007;2:46.

11. Yanardag H, Caner M, Papila I, et al. Diagnostic value of peripheral lymph node biopsy in sarcoidosis: A report of 67 cases. Can Respir J 2007;14:2209-116.

12. Sarkar M, Mahesh D, Madabhavi I. Digital clubbing. Lung India 2012;29:354.

13. Lieberman J. Elevation of serum angiotensin-convertingenzyme (ACE) level in sarcoidosis. Am J Med1975;59:365-72.

14. Baughman RP, Teirstein AS, Judson MA, et al. A Case Control Etiologic Study of Sarcoidosis (ACCESS) research group. Clinical characteristics of patients in a case control study of sarcoidosis. Am J Respir Crit Care Med 2001;164:1885-9.

15. Ors F, Gumus S, Aydogan M, et al. HRCT findings of pulmonary sarcoidosis; relation to pulmonary function tests. Multidiscip Respir Med 2013;8:8.

16. Criado E, Sánchez M, Ramírez J, et al. Pulmonary sarcoidosis: Typical and atypical manifestations at high-resolution CT with pathologic correlation. RadioGraphics 2010;30:1567-86.

17. Murdoch J, Müller NL. Pulmonary sarcoidosis: changes on follow-up CT examination. Am J Roentgenol 1992;159:473-7.

18. Teirstein AS, Machac J, Almeida O, et al. Results of 188 whole-body fluorodeoxyglucose positron emission tomography scans in 137 patients with sarcoidosis. Chest 2007; 132:1949-53.

19. Silva L, Hertz F, Cruz D, et al. [Sarcoidose no sul do Brasil: estudo de 92 pacientes].[Article in Portuguese]. J Bras Pneumol 2005;31:398-406.
20. Darlington P, Gabrielsen A, Sörensson P, et al. Cardiac involvement in Caucasian patients with pulmonary sarcoidosis. Respir Res 2014;15:15.

21. Sulica R, Teirstein AS, Kakarla S, et al. Distinctive clinical, radiographic, and functional characteristics of patients with sarcoidosis-related pulmonary hypertension. Chest 2005;128:1483.

22. Mahapatra QS, Sahai K, Rathi KR, et al. Pulmonary sarcoidosis: An important differential diagnosis in transbronchial lung biopsies. Lung India 2014;31:139-41.

23. Soares RV, Forsythe A, Hogarth K, et al. Interstitial lung disease and gastroesophageal reflux disease: key role of esophageal function tests in the diagnosis and treatment. Arq Gastroenterol 2011;48:91-7.

24. Westney GE, Habib S, Quarshie A. Comorbid illnesses and chest radiographic severity in African-American sarcoidosis patients. Lung 2007;185:131-7.

25. Martusewicz-Boros MM, Boros PW, Wiatr E, RoszkowskiŚliż K. What comorbidities accompany sarcoidosis? A large cohort $(\mathrm{n}=1779)$ patients analysis. Sarcoidosis Vasc Diffuse Lung Dis 2015;32:115-20.

26. Turner GA, Lower EE, Corser BC, et al. Sleep apnea in sarcoidosis. Sarcoidosis Vasc Diffuse Lung Dis 1997;14:61-4.

27. Okumus G, Musellim B, Cetinkaya E, et al. Extrapulmonary involvement in patients with sarcoidosis in Turkey. Respirology 2011;16:446-50.

28. Chung YM, Lin YC, Liu YT, et al. Uveitis with biopsy-proven sarcoidosis in Chinese--a study of 60 patients in a uveitis clinic over a period of 20 years. J Chin Med Assoc 2007;70:492-6.

29. Crouser ED, Maier LA, Wilson KC, et al. Diagnosis and detection of sarcoidosis. An official American Thoracic Society Clinical Practice Guideline. Am J Respir Crit Care Med 2020;201:e26-51. 